\title{
Fatores associados ao comportamento alimentar inadequado em adolescentes escolares
}

\author{
Factors associated with inappropriate eating behavior in adolescent students \\ Leonardo de Sousa Fortes ${ }^{1}$, Fabiane Frota da Rocha Morgado², Maria Elisa Caputo Ferreira ${ }^{1}$
}

1 Faculdade de Educação Física e Desportos da Universidade Federal de Juiz de Fora (UFJF), Juiz de Fora, MG, Brasil.

2 Faculdade de Educação Física da Universidade Estadual de Campinas (Unicamp), Campinas, SP, Brasil.

Laboratório de Estudos do Corpo (Labesc) - Faculdade de Educação Física e Desportos da Universidade Federal de Juiz de Fora (UFJF).

Recebido: 2/7/2012 - Aceito: 3/2/2013

\begin{abstract}
Resumo
Contexto: Evidências demonstram que a prevalência do comportamento alimentar inadequado (CAI) entre jovens tem aumentado nos últimos anos. Parece que a insatisfação corporal (IC), a prática excessiva de exercício físico, a composição corporal, o nível econômico (NE), além da etnia, podem ser fatores de risco para o CAI. Objetivo: Associar IC, grau de comprometimento psicológico ao exercício (GCPE), adiposidade corporal (AC), estado nutricional (EN), NE e etnia ao CAI em adolescentes. Métodos: Participaram 362 jovens de ambos os sexos com idades entre 10 e 19 anos. Utilizou-se o Eating Attitudes Test para avaliar o CAI. O Body Shape Questionnaire e a Commitment Exercise Scale foram utilizados para mensurar IC e GCPE, respectivamente. Aferiram-se dobras cutâneas para classificar a AC segundo sexo. Peso e estatura foram aferidos para calcular-se o índice de massa corporal (IMC) e classificar o EN. O NE foi avaliado pelo Critério de Classificação Econômica Brasil. Aplicou-se questionário que buscava avaliar etnia, idade e sexo. Resultados: Os resultados evidenciaram associação do CAI com IC, GCPE e NE no sexo feminino ( $p<0,05)$. Já entre os meninos, IC, AC e EN estiveram associados ao CAI (p < 0,05). Conclusão: Concluiu-se que IC foi a principal preditora de riscos para o CAI.
\end{abstract}

Fortes LS, et al. / Rev Psiq Clín. 2013;40(2):59-64

Palavras-chave: Comportamento alimentar, adolescentes, imagem corporal, atividade física.

\begin{abstract}
Background: Evidence shows that the prevalence of inappropriate eating behavior (CAI) among young people has increased in recent years. It seems that body dissatisfaction (IC), the practice of excessive exercise, body composition, the socioeconomic status (NE), and ethnicity may be risk factors for CAI. Objective: To associate IC, degree of psychological impairment to exercise (GCPE), adiposity (AC), nutritional status (EN), NE and ethnicity with CAI in adolescents. Methods: Participants 362 young people of both sexes aged between 10 and 19 years. We used the Eating Attitudes Test to assess the CAI. The Body Shape Exercise Questionnaire and Commitment Exercise Scale were used to measure IC and GCPE, respectively. We evaluated skinfold thickness is to classify the AC second sex. Weight and height were measured to calculate the index of mass (BMI) and classify the EN. The NE was evaluated by Brazil Economic Classification. Questionnaire was used that sought to assess ethnicity, age and sex. Results: The results showed association of CAI with IC, GCPE and NE in females (p < 0.05). Among boys, IC, AC and EN were associated with CAI ( $\mathrm{p}<0.05)$. Discussion: It was concluded that IC was the main predictor of risk for the CAI.
\end{abstract}

Fortes LS, et al. / Rev Psiq Clín. 2013;40(2):59-64

Keywords: Eating behavior, adolescents, body image, physical activity.

\section{Introdução}

Adolescência é caracterizada pela faixa etária dos 10 aos 19 anos ${ }^{1}$. Nesse período, o jovem vivencia alterações no âmbito social, psicológico e morfológico ${ }^{2}$. No entanto, essas modificações apresentam variabilidade no tempo, duração e intensidade entre os indivíduos. Meninas, por exemplo, costumam atingir o pico de velocidade de crescimento em estatura aos 12 anos, enquanto o sexo masculino geralmente chega a esse evento maturacional por volta dos 14 anos de idade ${ }^{3}$.

Alguns registros apontam que jovens escolares podem estar mais vulneráveis para modularem sentimentos e comportamentos a respeito do próprio corpo durante essa fase ${ }^{4-6}$. Esse fato tem sido atribuído a possível influência negativa de pais e amigos que costumam pressionar adolescentes a perder peso e investir em sua aparência física, podendo predispor esses jovens ao desenvolvimento de hábitos alimentares inadequados 7,8 .

Os comportamentos alimentares inadequados são hábitos deletérios à saúde desenvolvidos na tentativa de perda/controle de peso corporal ${ }^{9,10}$. Entre eles, pode-se destacar: vômitos autoindu- zidos, restrição patológica alimentar, compulsão alimentar, uso de medicamentos para emagrecimento (diuréticos, laxantes e pílulas dietéticas), entre outros ${ }^{11}$. Dadas suas características, esses comportamentos fazem parte dos critérios diagnósticos para os transtornos alimentares como anorexia nervosa $(\mathrm{AN})$ e bulimia nervosa $(\mathrm{BN})^{12}$.

Existem evidências na literatura de que a prevalência dessas atitudes tem aumentado entre adolescentes de ambos os sexos nos últimos anos ${ }^{5-6,10,13,14}$. Particularmente, entre meninos, essa constatação é considerada recente, enquanto, com o público feminino, os estudos nessa área têm sido realizados desde a década de $1980^{15}$. Todavia, com o masculino, somente nos últimos anos tem aumentado o interesse em pesquisar os comportamentos alimentares inadequados $\mathrm{e}$, em conjunto com esse interesse, tem sido constatado o aumento da frequência desses hábitos ${ }^{16,17}$.

Quanto aos fatores considerados de risco para o desencadeamento de hábitos alimentares inadequados em adolescentes, a literatura sugere alguns, quais sejam: a) insatisfação corporal - considerada um desgosto profundo com o corpo $0^{5,11,18}$; b) prática extenuante de atividade física ${ }^{19}$; c) estado nutricional inadequado ${ }^{20}$; d) adiposidade corporal elevada ${ }^{6}$, etnia branca ${ }^{10}$; e e) estrato econômico elevado ${ }^{7,21}$. 
Entretanto, até o momento, não temos o conhecimento de estudos desenvolvidos no Brasil com o propósito de associar a prática excessiva de exercício físico com os hábitos alimentares inadequados. Além disso, a maioria das pesquisas tem sido desenvolvida utilizando amostras somente do sexo feminino. Portanto, variáveis afetivas e comportamentais não têm sido avaliadas com a mesma frequência entre os meninos.

Conhecer os fatores associados aos comportamentos alimentares inadequados em adolescentes escolares torna-se uma investigação de importância central aos profissionais da área da saúde, em especial, ao professor de Educação Física. Esse profissional poderia ficar atento às atitudes de seus alunos em escolas, academias e clubes e propor estratégias de intervenção mediante a observação de alguma atitude de risco ao comportamento alimentar inadequado. Ademais, estender esse conhecimento ao público masculino poderia preencher possíveis "lacunas" na literatura dessa área, proporcionando a esse público um atendimento mais adequado às suas reais necessidades.

Diante do pressuposto, o objetivo do presente estudo foi associar insatisfação corporal, grau de comprometimento psicológico ao exercício, adiposidade corporal, estado nutricional, nível econômico e etnia ao comportamento alimentar inadequado em adolescentes de Juiz de Fora, Minas Gerais.

Por conseguinte, algumas hipóteses foram formuladas para ambos os sexos, levando-se em consideração o que a literatura costuma preconizar $^{4-7,20,21}$. Nesse sentido, esperava-se que: 1) jovens insatisfeitos com sua imagem corporal apresentassem maior chance para o desencadeamento dos comportamentos alimentares inadequados; 2) adolescentes com elevado grau de comprometimento psicológico ao exercício demonstrassem maiores riscos para o desenvolvimento de comportamentos alimentares inadequados; 3) escolares com morfologia considerada inadequada fossem evidenciados com chances aumentadas para os comportamentos alimentares inadequados; 4) jovens de estratos econômicos mais altos apresentassem elevados riscos para o desencadeamento de comportamentos alimentares inadequados; 5) e, por fim, escolares de etnia branca demonstrassem chances acentuadas para o desenvolvimento de comportamentos alimentares inadequados.

\section{Métodos}

Este estudo apresentou delineamento transversal e foi realizado no ano de 2011. Teve seu início após sua aprovação pelo Comitê de Ética e Pesquisa em Seres Humanos da Universidade Federal de Juiz de Fora, obtendo-se protocolo 2282.022.2011 e parecer número 022/11, de acordo com a resolução 196/96 do Conselho Nacional de Saúde.

\section{Sujeitos}

Os sujeitos desta pesquisa foram adolescentes, de ambos os sexos, com idades entre 10 e 15 anos, matriculados em instituições de ensino fundamental e médio da cidade de Juiz de Fora/MG. De acordo com o Censo Escolar de 2010, essa população era na ordem de 60 mil jovens. A amostra foi calculada por meio do método descrito por Miranda et al. ${ }^{4}$ e Field ${ }^{22}$, adotando-se uma precisão absoluta de dois pontos para mais ou para menos na variável resposta de referência, o escore do Eating Attitudes Test (EAT-26) ${ }^{15}$, e a maior variabilidade de pontuação verificada para o sexo feminino ( $\pm 12,9$ pontos $)^{6}$, como a pior situação ao nível de significância de $1 \%$, obteve-se um tamanho de amostra mínimo necessário igual a 276, já feita a correção para populações finitas. No entanto, 395 adolescentes participaram do estudo, porém 33 desses não responderam aos questionários em sua totalidade ou não participaram das aferições antropométricas, obtendo-se amostra final de 362 adolescentes (199 meninas e 163 meninos).

A amostra proporcional foi estratificada segundo a inserção das escolas nas regiões sociogeográficas do município de Juiz de Fora (norte, sul e centro) e o tipo de vinculação administrativa (pública e privada) e, em seguida, distribuída no ensino fundamental/médio. A seleção ocorreu aleatoriamente, por meio de sorteio simples, em duas etapas. Realizaram-se, primeiro, o sorteio das escolas em cada região e, posteriormente, o sorteio dos adolescentes nessas unidades. As escolas foram selecionadas valendo-se da relação fornecida pelo setor de estatística da Secretaria de Educação do Estado de Minas Gerais - Brasil. Desse modo, diretores de dez escolas (cinco privadas e cinco públicas) foram convidados a participar da pesquisa, sendo informados sobre objetivos e procedimentos do estudo. No entanto, somente seis desses (três privadas e três públicas) concordaram em liberar os alunos para participação nas coletas. Por fim, a amostra final da pesquisa foi distribuída em seis pontos diferentes de coleta (escolas) e constituída por adolescentes presentes nas escolas nos dias da coleta.

\section{Instrumentos}

Eating Attitudes Test em sua versão curta (EAT-26)23,24 - Utilizado para avaliar comportamentos alimentares inadequados. Trata-se de um instrumento autorreportado composto por 26 questões em escala do tipo Likert, variando de $0=$ nunca a $3=$ sempre, com exceção do item 25 , que apresenta pontuação invertida $(0=$ sempre, muitas vezes e às vezes; $1=$ poucas vezes; $2=$ quase nunca; 3 = nunca). Escore $\geq 20$ representa indivíduos com risco para o desenvolvimento de transtorno alimentar $\left(\mathrm{EAT}_{+}\right)$. As questões do EAT-26 buscam avaliar dieta, preocupação com forma física e com alimentos, sintomas de bulimia, pressão ambiental e social para ingesta alimentar, além de autocontrole oral. Neste estudo, duas versões do EAT-26 foram utilizadas: a) uma validada para adolescentes brasileiras do sexo feminino ${ }^{23}$ e a outra validada para adolescentes brasileiros do sexo masculino ${ }^{24}$. Ambas apresentaram consistência interna de 0,82 e 0,88 , respectivamente. Para a presente amostra, identificaram-se valores de alpha de Cronbach de 0,89 para meninas e 0,86 para meninos.

Body Shape Questionnaire (BSQ)25 - Utilizado para avaliar a insatisfação corporal. Esta ferramenta é constituída por 34 perguntas em escala do tipo Likert variando de $1=$ nunca até $6=$ sempre, que se propõem avaliar a frequência de preocupação com o peso e com a aparência física. O BSQ distingue os sujeitos a respeito de sua insatisfação corporal segundo o escore total ( $\geq 80=$ insatisfação corporal). Esse questionário foi validado para a população adolescente brasileira por Conti et al. ${ }^{25}$, apresentando boas propriedades psicométricas. Para a presente amostra, obtiveram-se valores de alpha de Cronbach de 0,92 e 0,90 para o sexo feminino e masculino, respectivamente.

Commitment Exercise Scale ${ }^{19}$ - Utilizada para determinar o grau de comprometimento psicológico que um indivíduo possa ter com o hábito de se exercitar. O instrumento foi traduzido, adaptado e validado para a língua portuguesa por Teixeira et al. ${ }^{19}$, sendo denominado Escala de Dedicação ao Exercício (EDE), que apresentou alpha de Cronbach de 0,79. A EDE avalia o grau com que sensações de bem-estar são moduladas pelo exercício, a manutenção do exercício em face de condições adversas e o grau de interferência que a atividade física tem em compromissos sociais do indivíduo. Trata-se de uma escala analógica visual, composta por oito questões que variam de 0 a $155 \mathrm{~mm}$ e, portanto, com uma pontuação máxima de $1.240 \mathrm{~mm}$. Escore maior que 640 indica sujeitos com elevado grau de comprometimento psicológico ao exercício $\left(\mathrm{EDE}_{+}\right)^{19}$. Calculou-se a consistência interna para a presente amostra, identificando-se alpha de Cronbach de 0,67 e 0,70 para o sexo feminino e masculino, respectivamente.

\section{Medida de outras variáveis}

Adiposidade corporal - Determinou-se a adiposidade corporal pelo percentual de gordura $(\% \mathrm{G})$. Esse percentual foi estimado pelo método duplamente indireto, mensurando-se as dobras cutâneas tricipital e subescapular. Utilizou-se o protocolo de Slaughter et al. ${ }^{26}$ para executar tal estimativa. Essas medidas foram realizadas de forma rotacional e coletadas três vezes, considerando-se a média dos valores. A classificação dessa variável foi atribuída pelos pontos 
de corte estabelecidos por Lohman ${ }^{27}$, que levam em consideração o sexo do sujeito.

Estado nutricional - $\mathrm{O}$ escore $\mathrm{z}$ do índice de massa corporal [IMC $=$ massa corporal $(\mathrm{kg}) /$ estatura $\left.\left(\mathrm{m}^{2}\right)\right]$ e da estatura/idade foi usado como indicador de estado nutricional. A classificação do escore z do IMC obedeceu aos critérios da Organização Mundial de Saúde (OMS) ${ }^{1}$, que propõe a classificação em baixo peso, peso normal, sobrepeso e obeso, de acordo com os escores $\mathrm{z}(-2,+1 \mathrm{e}+2)$, segundo a idade cronológica. Do mesmo modo, a normatização do escore $\mathrm{z}$ da estatura/idade seguiu as regras estabelecidas pela OMS ${ }^{1}$, que sugere a classificação em muito baixa estatura, baixa estatura e estatura adequada, em função dos escores $\mathrm{z}(-3,-2 \mathrm{e}+1)$ segundo a idade cronológica. A massa corporal foi mensurada por uma balança digital portátil da marca Tanita com precisão de 100 g e capacidade máxima de $200 \mathrm{~kg}$. Utilizou-se estadiômetro portátil com precisão de $0,1 \mathrm{~cm}$ e altura máxima de 2,20 $\mathrm{m}$ da marca Welmy para mensurar a estatura dos adolescentes.

Nível econômico - Definido por meio do "Critério de Classificação Econômica Brasil"28 desenvolvido pela Associação Brasileira de Empresas de Pesquisa. O Critério de Classificação Econômica Brasil enfatiza sua função de estimar o poder de compra das pessoas e famílias urbanas, abandonando a pretensão de classificar a população em termos de "classes sociais". Esse instrumento avalia a quantidade de itens de conforto (automóvel, geladeira, televisão etc.) adquiridos, além de identificar o grau de instrução do chefe de família. Ele nos remete aos seguintes pontos de corte em relação à classe econômica: A 1 - 30 a 34 pontos; A2 - 25 a 29; B1 - 21 a 24; B2 - 17 a 20; C - 11 a $16 ; \mathrm{D}-6$ a $10 ; \mathrm{E}-0$ a 5 .

Etnia - Definida pelo autorreporte dos alunos, os quais puderam escolher entre três opções de resposta: branco, negro ou amarelo. Esse modo de avaliar etnia foi inspirado nos estudos de Pernick et al. ${ }^{13}$ e Sampei et al. ${ }^{10}$.

\section{Procedimento de coleta de dados}

A priori, procurou-se a direção de cada escola participante do presente estudo para explanar os objetivos e procedimentos da pesquisa. Após autorização concebida, os pesquisadores se dirigiram aos alunos de cada turma para informar sobre os procedimentos de coleta de dados e entregar o Termo de Consentimento Livre e Esclarecido, pedindo-lhes que devolvessem devidamente assinados pelos responsáveis na semana subsequente a esse encontro.

Os dados foram coletados entre os meses de março e junho de 2011, com mínimo de dois momentos. No primeiro, em sala de aula, após a entrega do TCLE devidamente assinado pelos responsáveis, os alunos responderam individualmente aos questionários que continham cabeçalhos de identificação contendo nome (opcional), sexo, idade e etnia. Com a entrega dos questionários (segundo momento), o aluno foi encaminhado para uma sala cedida pela escola para mensuração de dobras cutâneas, peso e estatura. Para tanto, esse jovem deveria estar trajando uniforme para a aula de Educação Física e descalço.

\section{Análise dos resultados}

Os resultados foram avaliados com procedimentos estatísticos multivariados. Utilizou-se o software SPSS versão 17.0, adotando-se nível de significância de 5\%. Adotaram-se medidas de tendência central (média e desvio-padrão) e frequências (absoluta e relativa) para descrever as variáveis da pesquisa. Conduziu-se regressão logística binária para associar as variáveis independentes (insatisfação corporal, grau de comprometimento psicológico ao exercício, adiposidade corporal, estado nutricional, nível econômico e etnia) ao comportamento alimentar inadequado. Para isso, foi necessário agrupar-se as classificações de insatisfação corporal do BSQ em "insatisfeitos"; aglutinarem-se as classificações "muito baixo" e "baixo" da adiposidade corporal em "baixo", além de juntar os grupos "moderadamente alto", "alto" e "muito alto" de adiposidade corporal em "alto". Ademais, os subgrupos "sobrepeso" e "obeso" foram agrupados em "excesso de peso" e os estratos econômicos (ABEP) foram denominados A (A1 e A2), B (B1 e B2) e C (C, D e E).

\section{Resultados}

Ao todo, participaram do estudo 362 jovens de ambos os sexos, sendo 199 meninas e 163 meninos, com médias de $12,73( \pm 1,59)$ e 12,65 $( \pm 1,69)$ anos para o sexo feminino e masculino, respectivamente. Esses adolescentes eram pertencentes a seis instituições de ensino fundamental/médio da cidade de Juiz de Fora/MG, três privadas e três públicas. Medidas de tendência central (média e desvio-padrão) de todas as variáveis da pesquisa (EAT-26, BSQ, EDE, adiposidade corporal [\%G], estado nutricional [IMC], nível econômico [ABEP] e idade) e distribuição de frequência de etnia estão elucidadas na tabela 1. Os achados apontaram médias superiores para os escores do EAT-26, do BSQ e o \%G no sexo feminino em relação aos meninos. Por outro lado, os resultados indicaram valores de tendência central maiores no EDE para o sexo masculino. Todavia, as médias do IMC e do ABEP foram semelhantes entre os sexos. Por fim, grande parte dos participantes da presente investigação era de etnia branca.

Em relação à razão de chances para o comportamento alimentar inadequado no sexo feminino, o modelo de regressão logística ajustada para todas as variáveis demonstrou associação com insatisfação corporal, grau de comprometimento psicológico ao exercício e nível econômico $(\mathrm{p}<0,05)$ (Tabela 2$)$. Já no sexo masculino, insatisfação corporal, adiposidade corporal e estado nutricional (IMC) estiveram associados ao comportamento alimentar inadequado $(\mathrm{p}<0,05)$, como pode ser observado na tabela 2 .

\section{Discussão}

O presente estudo buscou associar insatisfação corporal, grau de comprometimento psicológico ao exercício, adiposidade corporal, estado nutricional, nível econômico e etnia ao comportamento alimentar inadequado em adolescentes de Juiz de Fora, Minas Gerais.

Os resultados da presente pesquisa no sexo feminino corroboram alguns aspectos de outros achados ${ }^{7,14,21}$. Nos referidos achados, encontraram-se 19,44 vezes mais chances de meninas insatisfeitas com o próprio corpo desenvolverem atitudes alimentares inadequadas. Parece que a insatisfação corporal é a principal variável que reflete riscos para o desenvolvimento do comportamento alimentar inadequado entre jovens do sexo feminino $5,6,18$.

Tabela 1. Descrição (média, desvio-padrão ou frequência) das variáveis do estudo. Juiz de Fora, 2011

\begin{tabular}{|c|c|c|}
\hline \multirow{3}{*}{ Variável } & \multicolumn{2}{|c|}{ Sexo } \\
\hline & Feminino $(n=199)$ & Masculino $(n=163)$ \\
\hline & \multicolumn{2}{|c|}{ Média (desvio-padrão) } \\
\hline EAT-26 & $15,08( \pm 11,18)$ & $11,49( \pm 11,46)$ \\
\hline BSO & $79,96( \pm 35,29)$ & $62,81( \pm 28,33)$ \\
\hline EDE & $498,18( \pm 215,53)$ & $548,66( \pm 218,49)$ \\
\hline$\% \mathrm{G}$ & $25,66( \pm 7,27)$ & $21,74( \pm 10,26)$ \\
\hline $\mathrm{IMC}\left(\mathrm{kg} / \mathrm{m}^{2}\right)$ & $19,77( \pm 3,66)$ & $19,97( \pm 3,51)$ \\
\hline ABEP & $18,93( \pm 4,89)$ & $19,06( \pm 4,59)$ \\
\hline \multirow[t]{2}{*}{ Idade } & $12,73( \pm 1,59)$ & $12,65( \pm 1,69)$ \\
\hline & \multicolumn{2}{|c|}{$\mathrm{N}(\%)$} \\
\hline \multicolumn{3}{|l|}{ Etnia } \\
\hline Branco & $134(67,3 \%)$ & $117(71,8 \%)$ \\
\hline Negro & $48(24,2 \%)$ & $35(21,5 \%)$ \\
\hline Amarelo & $17(8,5 \%)$ & $11(6,7 \%)$ \\
\hline
\end{tabular}

EAT-26: Eating Attitudes Test; BSQ: Body Shape Questionnaire; EDE: Escala de Dedicação ao Exercício; \%G: Percentual de Gordura; IMC: IIndice de Massa Corporal; ABEP: Critério de Classificação Econômica Brasil. 
Tabela 2. Razão de chances para comportamento alimentar inadequado (categoria de referência: EAT < 20) em adolescentes segundo insatisfação corporal, grau de comprometimento psicológico ao exercício, adiposidade corporal, estado nutricional, nível econômico e etnia. Juiz de Fora, 2011

\begin{tabular}{|c|c|c|c|c|c|}
\hline \multirow{2}{*}{ Variável } & \multirow{2}{*}{ Classificação } & \multicolumn{2}{|c|}{ Feminino } & \multicolumn{2}{|c|}{ Masculino } \\
\hline & & $\mathrm{OR}^{*}$ & IC (95\%) & $O R^{*}$ & IC $(95 \%)$ \\
\hline \multirow[t]{2}{*}{$\mathrm{BSO}_{\mathrm{a}, \mathrm{b}}$} & Satisfeito & 1,00 & - & 1,00 & - \\
\hline & Insatisfeito & 19,44 & $7,66-31,88$ & 7,97 & $2,56-24,81$ \\
\hline \multirow[t]{2}{*}{ EDEa } & Negativo & 1,00 & - & 1,00 & - \\
\hline & Positivo (EDE+) & 2,14 & $1,05-5,41$ & 0,94 & $0,32-2,75$ \\
\hline \multirow[t]{3}{*}{$\% G^{b}$} & Baixo & 0,67 & $0,08-5,29$ & 4,16 & $3,56-28,74$ \\
\hline & Normal & 1,00 & - & 1,00 & - \\
\hline & Alto & 1,42 & $0,48-4,24$ & 5,77 & $3,66-20,26$ \\
\hline \multirow[t]{3}{*}{ ENo (IMC) } & Baixo peso & 1,29 & $1,50-13,88$ & 5,95 & $1,41-12,71$ \\
\hline & Peso normal & 1,00 & - & 1,00 & - \\
\hline & Excesso de peso & 1,93 & $0,56-8,13$ & 1,78 & $1,06-9,92$ \\
\hline \multirow[t]{3}{*}{ EN (E/I) } & Muito baixa & 1,06 & $0,55-2,76$ & 1,23 & $0,95-3,04$ \\
\hline & Baixa & 0,87 & $0,61-3,97$ & 1,14 & $0,89-4,23$ \\
\hline & Adequada & 1,00 & - & 1,00 & - \\
\hline \multirow[t]{3}{*}{ NEa } & A & 1,16 & $1,04-3,10$ & 1,15 & $0,36-3,68$ \\
\hline & B & 3,94 & $1,99-15,65$ & 0,69 & $0,09-5,06$ \\
\hline & C & 1,00 & - & 1,00 & - \\
\hline \multirow[t]{3}{*}{ Etnia } & Branco & 0,83 & $0,31-2,22$ & 1,11 & $0,31-3,97$ \\
\hline & Negro & 1,00 & - & 1,00 & - \\
\hline & Amarelo & 0,84 & $0,13-5,64$ & 0,10 & $0,01-4,25$ \\
\hline
\end{tabular}

BSQ: Body Shape Questionnaire; EDE: Escala de Dedicação ao Exercício; \%G: percentual de gordura; EN: estado nutricional; IMC: índice de massa corporal; E/l: estatura/idade; NE: nível econômico; $O R^{*}$ : razão de chances (odds ratio) ajustada para todas as variáveis; IC: intervalo de confiança. a $p<0,05$ no sexo feminino; ${ }^{b} p<0,05$ no sexo masculino.

Em adição, este estudo demonstrou que meninas com pontuações acima de 640 no $\mathrm{EDE}\left(\mathrm{EDE}_{+}\right)$, ou seja, aquelas com elevado grau de comprometimento psicológico ao exercício, apresentaram 2,14 vezes mais chances de alimentação transtornada quando comparadas a jovens com pontuações menores de 640 . Todavia, não se encontraram outros estudos com esse tipo de análise que fosse passível de comparações. No entanto, pesquisadores afirmam que sujeitos com comportamentos alimentares de risco para transtornos alimentares costumam praticar exercícios físicos em excesso ${ }^{29}$.

Desse modo, acredita-se que jovens do sexo feminino que praticam exercícios de forma exacerbada tenham tais hábitos na tentativa de reduzirem os sentimentos depreciativos com o próprio corpo ${ }^{12,29}$. No entanto, parece que esse fenômeno acontece em forma de "cascata", ou seja, meninas insatisfeitas com o corpo buscam a prática de atividade física almejando remodelamento corporal ${ }^{4,30}$. Entretanto, essas podem aumentar os riscos para o desencadeamento de condutas alimentares deletérias à saúde caso tenham grande comprometimento para tal prática ${ }^{29}$. Uma possível explicação para esse fato foi focalizada por Teixeira et al. ${ }^{12}$. Os autores afirmam que jovens do sexo feminino com hábitos alimentares inapropriados tendem maximizar a perda de peso corporal, despendendo seus esforços em atividades físicas extenuantes.

Neste estudo, não se identificou associação nem da adiposidade corporal nem do estado nutricional com o comportamento alimentar inadequado no sexo feminino. Entretanto, resultados de outros estudos demonstraram que meninas com elevada adiposidade corporal e/ou classificadas com sobrepeso/obesidade pelo IMC apresentaram riscos acentuados sobre os hábitos alimentares ${ }^{14,21}$. Apesar de não se achar associação, parece que gordura corporal acentuada pode ser considerada fator de risco para a instalação dos comportamentos alimentares inadequados no sexo feminino ${ }^{5-6}$.

Ademais, evidenciaram-se 3,94 vezes mais chances de meninas com nível econômico " $\mathrm{B}$ ” desencadearem comportamentos compensatórios para controle de peso. Dunker et al. ${ }^{21}$ salientam que os hábitos alimentares deletérios à saúde são mais frequentes nos estratos econômicos mais altos da sociedade, contrapondo os achados deste estudo. Por outro lado, autores ressaltam que essa tendência é mais comum em grandes capitais ou regiões com elevado índice de desenvolvimento humano, podendo não ser válida para cidades menores ${ }^{14}$, como Juiz de Fora. Por fim, a etnia não apresentou associação com as classificações do EAT-26 no modelo de regressão logística binária. Em contrapartida, Sampei et al. ${ }^{10}$, em seus achados, demonstraram que jovens caucasianas (brancas) apresentaram 2,43 vezes mais chances de desenvolverem atitudes alimentares de risco para transtornos alimentares. Portanto, acredita-se que a etnia ainda permaneça como tópico impreciso a respeito de riscos para o comportamento alimentar inadequado.

Em relação ao sexo masculino, são raros os estudos que avaliaram a possibilidade de associação do comportamento alimentar inapropriado com fatores considerados de risco para tal desfecho, dificultando, então, as comparações dos resultados. Os achados da presente pesquisa para esse sexo evidenciaram que jovens insatisfeitos com peso e aparência física apresentaram maiores riscos para o desenvolvimento de hábitos alimentares não saudáveis (7,97 vezes mais chances). Autores ressaltam que, assim como no sexo feminino, entre os meninos a insatisfação corporal parece ser a principal variável desencadeadora de atitudes alimentares inadequadas ${ }^{4,20}$. No que diz respeito ao grau de comprometimento psicológico ao exercício, 
este não apresentou associação significativa com a classificação EAT do EAT-26 no modelo de regressão logística. Parece que o comportamento alimentar inadequado neste sexo independe do excesso de atividade física ${ }^{29}$. Uma possível explicação é que a prática excessiva de exercício físico entre os meninos esteja mais especificamente atrelada ao ganho de massa muscular e não à restrição alimentar e/ ou aos sintomas de bulimia ${ }^{12}$.

Em contrapartida, a adiposidade corporal demonstrou associação com o comportamento alimentar inadequado no sexo masculino. Meninos classificados com percentual de gordura "baixo" e "alto" apresentaram 4,16 e 5,77 vezes mais chances para os hábitos alimentares deletérios à saúde, respectivamente, quando comparados aos jovens com adiposidade corporal dentro dos padrões considerados normais. Pesquisadores afirmam que adolescentes com perfil lipídico abaixo do normal costumam apresentar restrição da ingesta de alimentos como hábito alimentar; enquanto jovens com porcentagem de gordura elevada podem demonstrar sintomas de compulsão alimentar ${ }^{14,18}$. Assim como na adiposidade corporal, o estado nutricional, avaliado pelo IMC, também esteve associado com o desfecho no sexo masculino. Identificaram-se maiores riscos para o comportamento alimentar inadequado em indivíduos com baixo peso corporal ( 5,95 vezes mais chances). Todavia, o estado nutricional, avaliado pela estatura/idade, não demonstrou associação com o comportamento alimentar entre os meninos. De qualquer forma, os achados mencionados acima estão de acordo com as hipóteses levantadas por Johnson et al. ${ }^{9}$. Segundo esses autores, meninos com baixo peso costumam demonstrar maior tendência para restrição alimentar, preocupação com aparência física, além de estarem mais suscetíveis para influências negativas sociais sobre a ingestão alimentar.

Por outro lado, nível econômico e etnia não apresentaram associação com o fator positivo do EAT-26 entre os meninos. Autores salientam que jovens do sexo masculino costumam apresentar menores riscos para o comportamento alimentar, independente da classe econômica e da etnicidade ${ }^{9,13}$. Talvez, adolescentes do sexo masculino de diferentes níveis econômicos e distintas etnias sofram influências midiáticas semelhantes a respeito do ideal corporal.

O presente estudo demonstrou resultados relevantes para a literatura científica brasileira. Todavia, alguns resultados merecem ser interpretados com cautela. Utilizaram-se questionários autoaplicáveis como ferramentas de pesquisa. Autores ressaltam que questionários podem não refletir a realidade do contexto avaliado, pois se tratam de instrumentos autorreportados com respostas subjetivas ${ }^{11}$. Por outro lado, pesquisadores salientam que, em estudos com grandes amostras, os questionários são ferramentas mais viáveis para serem utilizadas, pois apresentam fácil manuseio e baixo custo operacional ${ }^{4}$. Acrescentando, a etnia foi avaliada por instrumento construído pelos próprios autores, o que poderia ser outra limitação da presente pesquisa, porém outros estudos utilizaram o mesmo método de avaliação para tal variável9,10,13. Finalmente, o desenho do tipo transversal não permitiu que fosse realizada inferência de causalidade. Isso significa que não se tem como avaliar o grau de intensidade e a direção das associações encontradas entre o desfecho do estudo e as variáveis independentes. Na realidade, essa é uma limitação do estudo transversal, por apresentar um retrato instantâneo do contexto pesquisado.

Por fim, concluiu-se que o comportamento alimentar inadequado esteve associado à insatisfação corporal e ao grau de comprometimento psicológico ao exercício no sexo feminino, corroborando as hipóteses levantadas na introdução. Todavia, os achados para o nível econômico nesse mesmo sexo contrapuseram tais hipóteses. Por outro lado, entre os meninos, somente a insatisfação corporal, a adiposidade corporal e o estado nutricional manifestaram associação com o desfecho do estudo. Nesse sentido, esses achados confirmaram as hipóteses formuladas no início dessa investigação.

Por conseguinte, recomenda-se que sejam realizados programas de intervenções multiprofissionais em escolas tanto da rede pública quanto privada de Juiz de Fora/MG, com o propósito de orientar os escolares a respeito de condutas alimentares corretas, hábitos cotidianos saudáveis, além da relação com o próprio corpo, visto que este se encontra em constante mudança durante a adolescência.

\section{Referências}

1. World Health Organization. Development of a WHO growth reference for school-aged children and adolescents. Bulletin of the World Health Organization. 2007;85(9):660-7.

2. Siervogel RM, Demerath EW, Schubert C, Remsberg KE, Chumlea WC, Sun S, et al. Puberty and body composition. Horm Research. 2003;60(1):36-45.

3. Machado DRL, Bonfim MR, Costa RT. Pico de velocidade de crescimento como alternativa para classificação maturacional associada ao desempenho motor. Rev Bras Cineantropom Desempenho Hum. 2009;11(1):14-21.

4. Miranda VPN, Conti MA, Bastos R, Ferreira MEC. Insatisfação corporal em adolescentes brasileiros de municípios de pequeno porte de Minas Gerais. J Bras Psiq. 2011;60(3):190-7.

5. Scherer FC, Martins CR, Pelegrini A, Matheus SC, Petroski EL. Imagem corporal em adolescentes: associação com a maturação sexual e sintomas de transtornos alimentares. J Bras Psiq. 2010;59(3):198-202.

6. Martins CR, Pelegrini A, Matheus SC, Petroski EL. Insatisfação com a imagem corporal e a relação com estado nutricional, adiposidade corporal e sintomas de anorexia e bulimia em adolescentes. Rev Psiq Rio Grande do Sul. 2010;32(1):19-23.

7. Vale AMO, Kerr RLS, Bosi MLM. Comportamento de risco para transtornos do comportamento alimentar entre adolescentes do sexo feminino de diferentes estratos sociais do Nordeste do Brasil. Ciênc Saúde Coletiva. 2011;16(1):121-32.

8. White J, Halliwell E. Examination of a sociocultural model of excessive exercise among male and female adolescents. Body Image. 2010;7(2):227-33.

9. Johnson C, Crosby R, Engel S, Mitchell J, Powers P, Wittrock D, et al. Gender, ethnicity, self-esteem and disordered eating among college athletes. Eating Behaviors. 2004;5(1):147-56.

10. Sampei MA, Singulem DM, Novo NF, Juliano Y, Colugnati FAB. Atitudes alimentares e imagem corporal em meninas adolescentes de ascendência nipônica e caucasiana em São Paulo (SP). J Ped. 2009;85(2):122-8.

11. Schaal K, Tafflet M, Nassif H, Thibault V, Pichard C, Alcotte M, et al. Psychological balance in high level athletes: gender-based differences and sport-specific patterns. PLoS One. 2011;6(5):e19007.

12. Teixeira PC, Costa RB, Matsudo SM, Cordás TA. A prática de exercícios físicos em pacientes com transtornos alimentares. Rev Psiq Clin. 2009;36(4):145-52.

13. Pernick Y, Nichols JF, Rauh MJ, Kern M, Ji M, Lawson MJ, et al. Disordered eating among a multi-racial/ethinic sample of female high school athletes. J Adol Health. 2006;38(4):689-95.

14. Alves E, Vasconcelos FAG, Calvo MCM, Neves J. Prevalência de sintomas de anorexia nervosa e insatisfação com a imagem corporal em adolescentes do sexo feminino do Município de Florianópolis, Santa Catarina, Brasil. Cad Saúde Pública. 2008;24(3):503-12.

15. Garner DM, Olmsted MP, Bohr Y, Garfinkel PA. The eating attitudes test: psychometric features and clinical correlations. Psycho Med. 1982;12(1):871-8.

16. Rivas T, Bersabé R, Jumenez M, Berrocal C. The eating attitudes test (EAT-26): reliability and validity in Spanish female samples. Span J Psychol. 2010;13(2):1044-56.

17. Silva L, Gomes AR, Martins C. Psychological factors related to eating disordered behaviors: a study with Portuguese athletes. The Spanish J Psycho. 2011;14(1):323-35.

18. Alvarenga MS, Scagliusi FB, Phillippi ST. Comportamento de risco para transtorno alimentar em universitárias brasileiras. Rev Psiq Clin. 2011;38(1):3-7.

19. Teixeira PC, Hearst N, Matsudo SMM, Cordás TA, Conti MA. Adaptação transcultural: tradução e validação de conteúdo da versão brasileira do Commitment Exercise Scale. Rev Psiq Clin. 2011;38(1):24-8.

20. Pelegrini A, Petroski EL. The association between body dissatisfaction and nutritional status in adolescent. Hum Mov. 2010;11(1):51-7.

21. Dunker KLL, Fernandes CPB, Filho DC. Influência do nível socioeconômico sobre comportamentos de risco para transtornos alimentares em adolescentes. J Bras Psiq. 2009;58(3):156-61.

22. Field A. Descobrindo a estatística usando o SPSS. Porto Alegre: Artmed; 2009 .

23. Bighetti F, Santos CB, Santos JE, Ribeiro RPP. Tradução e avaliação do Eating Attitudes Test em adolescentes do sexo feminino de Ribeirão Preto, São Paulo. J Bras Psiq 2004;53(6):339-46. 
24. Fortes LS, Amaral ACS, Conti MA, Cordás TA, Ferreira MEC. Qualidades psicométricas do Eating Attitudes Test (EAT-26) para a população adolescente masculina brasileira. Psicologia: Reflexão e Crítica. No prelo.

25. Conti MA, Cordás TA, Latorre MRDO. Estudo de validade e confiabilidade da versão brasileira do Body Shape Questionnaire (BSQ) para adolescentes. Rev Bras Saude Mater Infant. 2009;9(3):331-8.

26. Slaughter MH, Lohman TG, Boileau R, Hoswill CA, Stillman RJ, Yanloan MD. Skinfold equations for estimation of body fatness in children and youth. Hum Biology. 1988;60(3):709-23.
27. Lohman TG. The use of skinfolds to estimate body fatness on children and youth. J Phis Education Recreational Dance. 1987;58(1):98-103.

28. Associação Brasileira de Empresas de Pesquisa (ABEP). Critério de Classificação Econômica Brasil. Acessado em: 10/7/2009.

29. Modolo VB, Antunes HKM, Gimenez PRB, Santiago MLM, Tufik S, Mello MTM. Negative addiction to exercise: are there differences between genders? Clinics. 2011;66(2):255-60.

30. Nascimento AL, Appolinário, JC, Fontenelle LF. Comorbidade entre transtorno dismórfico corporal e bulimia nervosa. Rev Psiq Clin. 2012;39(1):40-2.

\section{O CONTEÚDO DA RPC TAMBÉM DISPONÍVEL NA VERSÃO iPad}

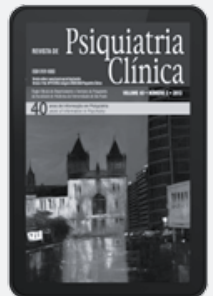

Para obter o aplicativo da RPC, é simples:

1. Entre na Apple Store via iPad.

2. Acesse a categoria "medicina".

3. Procure por" Psiquiatria Clínica" no campo de busca acima, à direita.

4. Faça o download gratuitamente. 